\title{
Stochastic Approach to a Rain Attenuation Time Series Synthesizer for Heavy Rain Regions
}

\author{
Masoud Mohebbi Nia ${ }^{1}$, Jafri Din ${ }^{1}$, Hong Yin Lam ${ }^{2}$, Athanasios D. Panagopoulos ${ }^{3}$ \\ ${ }^{1}$ Wireless Communication Center, Faculty of Electrical Engineering, 81310 Universiti Teknologi Malaysia, \\ Johor Bahru, Johor, Malaysia \\ ${ }^{2}$ Departement of Electrical Engineering Technology, Faculty of Engineering Technology, 86400, Universiti Tun Hussein \\ Onn Malaysia, Parit Raja, Johor Malaysia \\ ${ }^{3}$ School of Electrical and Computer Engineering, National Technical University of Athens, Zografou, Athen, Greece
}

\section{Article Info}

Article history:

Received May 16, 2016

Revised Jul 4, 2016

Accepted Jul 20, 2016

\section{Keyword:}

Heavy rain regions

Radiowave propagation

Rain attenuation

Stochastic approach

Time series synthesizer

\begin{abstract}
In this work, a new rain attenuation time series synthesizer based on the stochastic approach is presented. The model combines a well-known interestrate prediction model in finance namely the Cox-Ingersoll-Ross (CIR) model, and a stochastic differential equation approach to generate a longterm gamma distributed rain attenuation time series, particularly appropriate for heavy rain regions. The model parameters were derived from maximumlikelihood estimation (MLE) and Ordinary Least Square (OLS) methods. The predicted statistics from the CIR model with the OLS method are in good agreement with the measurement data collected in equatorial Malaysia while the MLE method overestimated the result. The proposed stochastic model could provide radio engineers an alternative solution for the design of propagation impairment mitigation techniques (PIMTs) to improve the Quality of Service (QoS) of wireless communication systems such as $5 \mathrm{G}$ propagation channel, in particular in heavy rain regions.
\end{abstract}

Copyright (C) 2016 Institute of Advanced Engineering and Science. All rights reserved.

Corresponding Author:

Jafri Din,

Wireless Communication Center, Faculty of Electrical Engineering,

Universiti Teknologi Malaysia,

81300, Skudai, Johor.

Email: jafri@utm.my

\section{INTRODUCTION}

Current and future wireless communication systems such as 5G cellular networks and satellite communication system are moving toward higher speed data rates and wider bandwidths with the employment of operating frequencies above $10 \mathrm{GHz}$ [1]. Unfortunately, radio link operating at these frequencies suffer strong attenuation phenomena due to the atmospheric constituents such as rain, cloud, water vapour and turbulent fluctuations/scintillation [2]-[5]. Among these, rain appears as the major factor that degrades the performance of wireless communication systems [2]. This impairment is even worse in heavy rain regions where the precipitation characteristics are significantly different from those in temperate areas [6]-[8]. In order to counteract such impairments that depend on the local climatology, detailed dynamic characteristics of precipitation are required to serve as the critical input to the advanced propagation impairment mitigation techniques (PIMTs) [2]. A signal measured directly from the communication system is the best resource for this purpose. However, such measured signals are not widely available; therefore, the propagation community proposed the synthetic rain attenuation time series to mimic the dynamic of real signals.

For the past decade, various studies have focused on the development of rain attenuation time series synthesizers [9]-[11]. Eventually, in 2009, International Telecommunication Union-Radio communication (ITU-R) recommended a stochastic approach to generate rainy and clear-sky time series [12]. Since then, 
several extended works have continued to improve on this series and proposed more refined synthesized time series. Carrie et al. extended the ITU-R model to an event-on-demand synthesizer in order to generate time series according to the demand of maximum attenuation level and event duration [13]. Boulanger et al. proposed a new time series synthesizer based on the combination of Dirac and lognormal distribution [14]. While in tropical areas, Andrade et al. proposed a model based on the gamma distribution where the model parameters were inferred from the real measured data collected in Brazil [15] and Kanellopoulos et al. presented a new stochastic dynamic model for the generation of rain attenuation time series based on the powerful solution of first-order stochastic differential equation (SDE) for the long-term Markov process [16].

In this work, we proposed an alternative and promising stochastic approach to synthesizing longterm rain attenuation time series, mainly focusing on areas that exhibit extreme heavy rainfall. The model presented here is an extended application of a well-established long-term interest rate generation model in financial research [17]. The Cox-Ingersoll-Ross model, hereinafter named "CIR", reproduces the dynamic characteristics of local precipitation with respect to the precipitation-attenuation phenomena.

The paper is organized as follows. First, we present the concept and principles of the CIR stochastic model as a rain attenuation time series synthesizer. General solutions and dynamic parameter computations of the model are explained in Section 3, followed by a brief discussion on the long term statistics of this model in Section 4. Section 5 present the numerical results validated against measured data in equatorial Malaysia and finally Section 6 draws some conclusions.

\section{STOCHASTIC MODEL OF TIME SERIES SYNTHESIZER}

In this work, the long-term complementary cumulative distribution function of rain attenuation is assumed to be gamma distribution as this is the well-established statistical distribution in heavy rain regions [16]. Hence, such distribution of satellite and terrestrial links can be described by a first-order stochastic differential equation $(\mathrm{SDE})$ :

$$
d X_{t}=D_{1}\left(X_{t}\right) d t+D_{2}\left(X_{t}\right) d W_{t}
$$

where $D_{1}$, and $D_{2}$ are drift and diffusion coefficients respectively representing slow and rapid varying components of the rain attenuation time series [18]. $d W_{t}$ is the Brownian motion or Wiener process that follows the normal distribution with zero mean and variance $d t$ [18],[19]. In this particular application, a satellite/terrestrial channel usually considered radio signals appearing in the form of additive white Gaussian noise (AWGN), hence the variance of the Wiener process can be assumed to be equal to 1 while $X_{t}$ represents the rain attenuation time series. Hence $d t$ is equal to 1 .

Based on these properties of distribution, the CIR model that is capable of generating a time series of interest rate over a long duration (i.e. more than 10 years) according to gamma distribution is clearly one of the best alternative tools to predict rain attenuation time series. This well-established model in finance and economics represents the first-order SDE as follows [17]:

$$
d X_{t}=k\left(\mu-X_{t}\right) d t+\sigma \sqrt{X_{t}} d W_{t}
$$

where $k, \mu$ and $\sigma$ are the three empirical parameters that could be inferred from the experimental rain attenuation dataset as a function of the transmission/receiving links elevation angle, polarization as well as operating frequency. It is also worth noting that $k$ corresponds to the dynamic factor $\beta$ of rain attenuation as clearly explained in [20]. Nevertheless, in the absence of experimental databases, several tools developed by propagation researchers such as synthetic storm techniques which only require the input of time series rain rate measured by local rain gauges, could also provide sufficient accuracy for the extraction of those parameters [6],[21]. The next section briefly discusses the general solution and methodology inferred by the CIR model parameters.

\section{CIR MODEL PARAMETERS ESTIMATION AND GENERAL SOLUTION}

One relevant issue for the modelling of a rain attenuation time series synthesizer is the long term distribution of first order rain attenuation statistics. In particular, these statistics in heavy rain regions seem to follow gamma distribution as observed in several previous works [15],[16]. For this reason, since the CIR model is an ergodic process with a stationary distribution, as the dynamic parameter $\mathrm{k}$ approaches the long term mean value of rain attenuation, the synthesized rain attenuation will approach gamma distribution. 
In general, since rain attenuation over the period of time can be described by the Weiner process (Brownian motion), Ito's process allows for the solution of the differential of a time-dependent function of rain attenuation process by the well-known approximation method Euler-Maruyama [18],[19]. EulerMaruyama approximation could provide the simplest yet strong Taylor approximation series as

$$
\begin{aligned}
& Y_{n+1}=Y_{n}+k Y_{n} \Delta_{n}+\xi Y_{n} \Delta W_{n} \\
& \Delta W_{n}=W_{\tau_{n}+1}-W_{\tau_{n}} \\
& \Delta_{n}=E\left(\left(\Delta \mathrm{W}_{n}\right)^{2}\right)
\end{aligned}
$$

To simulate a predicted rain attenuation time series for $n=0,1,2, \ldots N-1$, we simply start from the initial condition $Y_{o}=X_{o}$ and proceed to the next values. This is an Ito process with drift $k$ and diffusion $\xi$ coefficient.

After considering the theoretical estimation, two different techniques could be practically deployed to predict the SDE model parameters namely the Maximum Likelihood Estimation (MLE) [22] and the Ordinary Least Square (OLS). MLE is commonly employed to estimate multistep ahead forecasts. This approach determines the model parameters based on the most likely probability from the measured dataset. On the other hand, OLS estimates the model parameter from a linear regression by means of minimizing the difference between the measured data and the estimated values [23]. Simulation results of both approaches are subsequently shown in Section 5.

\section{GENERATION OF FIRST ORDER LONG-TERM RAIN ATTENUATION STATISTICS}

Before we further analyse the simulation results, it is worth discussing the reliability of long-term first-order rain attenuation statistics produced by the CIR model. In the planning and design of a wireless communication system, a reliable long-term CCDF of rain attenuation is of the utmost importance [24]. In order to regenerate such long-term statistics, the rain attenuation is usually described by a relative simple first-order Markov process [9],[16]. Hence it is best to represent the statistics in the form of the OrnsteinUhlenbeck process [19]. This is the only stochastic process that satisfies the properties of stationary, Gaussian and Markov processes.

The CIR model assumes that if the observation period $t$ is long enough $(t \rightarrow \infty)$, and the rain attenuation approaches the mean values of long-term observation data $\mathrm{m}$, the model can be simplified, by replacing $X_{t}$ with $m$ :

$$
d X_{t}=k\left(\mu-X_{t}\right) d t+\sigma \sqrt{m} d W_{t}
$$

This is also known as the Vasicek model [25] which was previously introduced to describe interest rate movement in finance and is now adapted to describe the dynamics of rain attenuation levels. The general solution of the SDEs with homogeneous coefficients and additive noise can now be written as:

$$
X_{t}=x_{o}+\mu\left(1-e^{k t}\right)+\sigma \sqrt{m} \int_{0}^{t} e^{k s} d W_{s}
$$

The integral of the Weiner process can be solved using Euler-Maruyama approximation from the long-term measurement data. The mean $\mu$ and variance $\sigma$ of the time series synthesizer from (7), which deals with the Brownian/ Wiener process, can be solved by using Itō integral from Itō lemma as [18],[19]:

$$
\begin{aligned}
& \mu=x_{o} e^{-k t}+\mu\left(e^{-k t}-1\right) \\
& \sigma^{2}=\frac{m \sigma^{2}}{2 k} e^{-2 k t}
\end{aligned}
$$




\section{NUMERICAL RESULTS AND DISCUSSION}

In this section, we present several numerical results from the CIR model and tested against one year of measurement database recorded in the Universiti Teknologi Malaysia campus, Skudai, Johor $\left(1.55^{\circ} \mathrm{N}\right.$, $\left.103.64^{\circ} \mathrm{E}\right)$ as illustrated in Figure 1. The ground station is looking toward the MEASAT-1 satellite $\left(91.5^{\circ} \mathrm{E}\right)$ with an elevation angle of $75.61^{\circ}$. The operating frequency is $11.075 \mathrm{GHz}$. For the sake of brevity, details of this measurement are clearly explained in [26] and will not be repeated here.

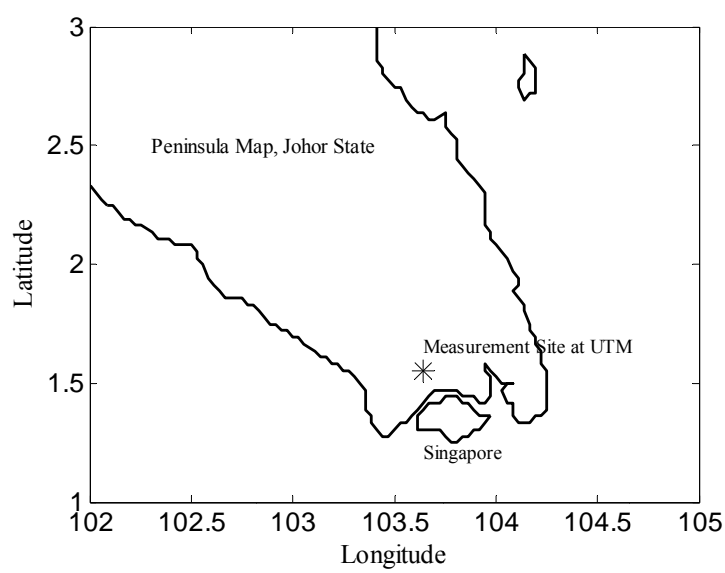

Figure 1. Map of the Malaysian peninsula showing the measurement site near an equatorial heavy rain region

As previously mentioned, MLE and OLS play a crucial role in synthesizing the long-term time series of rain attenuation. To this aim, first, an example of time series of rain attenuation synthesized with the MLE and OLS approaches is illustrated in Figure 2. At first glance, both methods seem to reproduce the dynamic properties of rain attenuation in one specific time series rain event. Since the aim of the synthesizer is to generate typical rain attenuation time series with respect to the local climatological characteristics as well as geometrical and radio parameters of one particular communication link, we vierified the capability of the CIR model with the MLE and OLS approaches in probability density function (PDF). The PDFs of rain attenuation values generated by the MLE and OLS approaches are compared with the measured PDF as depicted in Figure 3. The OLS method seems to closely follow the measured attenuation threshold (for the range of $0.1 \mathrm{~dB}$ to $4 \mathrm{~dB}$ ) while the MLE method overestimates PDF of attenuation value less than $0.5 \mathrm{~dB}$.
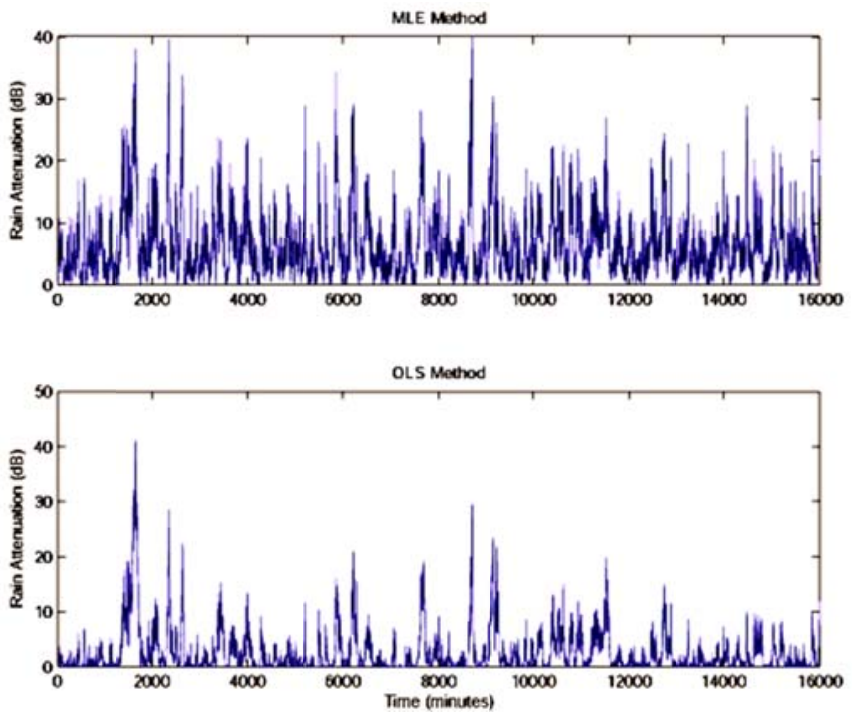

Figure 2. Example of synthesized rain attenuation time series by the CIR model with the MLE approach (Top) and the OLS approach (Bottom) for a ground station in UTM, Johor, Malaysia 


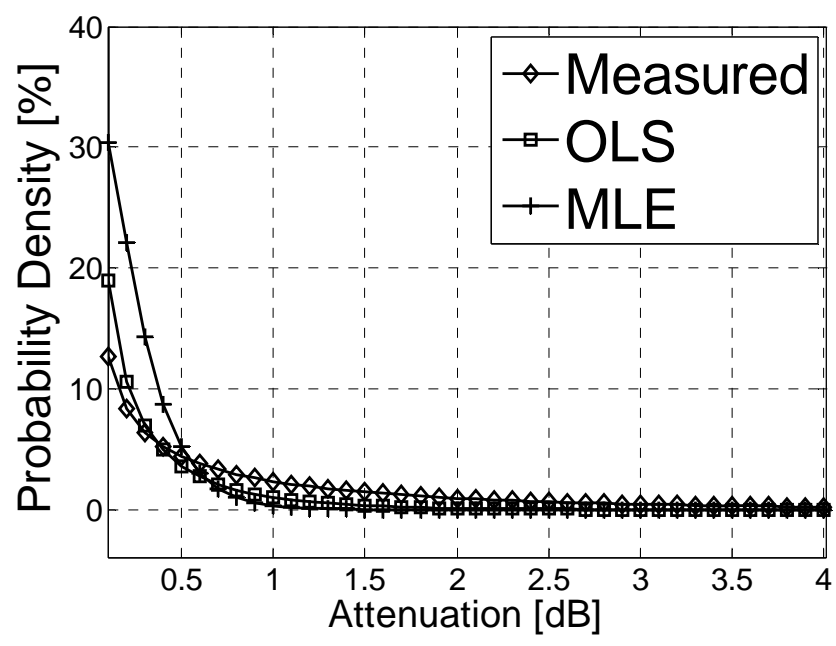

Figure 3. Probability density functions (PDFs) of attenuation threshold generated by the MLE and OLS approaches and compared with the measured PDF of the MEASAT satellite

In order to further evaluate the effectiveness of the CIR model from a propagation perspective, testing has been carried out by comparing long term CCDFs of rain attenuation between the CIR model (MLE), the CIR model (OLS) and the measured CCDF. The ITU-R Rec. P.618-12 [27] is also included in the comparison as this recommendation served as the main reference model for the prediction of first-order rain attenuation statistics. The results in Figure 4 clearly indicate the effectiveness of the CIR model with the OLS approach compared to the one with the MLE method. The OLS approach provides good agreement prediction with the measurement statistics while the MLE method marked an overestimation. On the other hand, ITU-R Rec. P.618 clearly underestimates the slant-path measured statistics, which highlights the importance of locally-derived parameters in providing statistics with better accuracy.

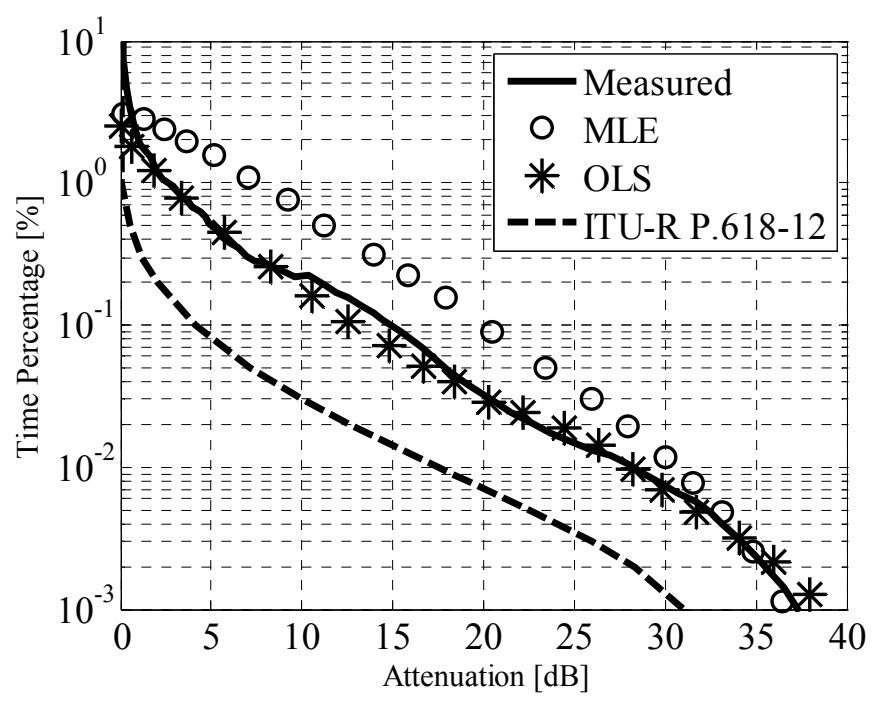

Figure 4. Comparison of Complementary cumulative distribution functions (CCDFs) of rain attenuation: MEASAT measured, the CIR model (MLE), the CIR model (OLS) and ITU-R recommendation

Figure 5 subsequently shows the error of the prediction with respect to the time percentage from $0.001 \%$ to $1 \%$. As can be seen, prediction errors of the MLE approach are obviously higher than those predicted by the OLS method. This can be ascribed to the shortcoming of the MLE method in that it does not accurately describe the density of each attenuation threshold (see Figure 3). For these reasons, as clearly mentioned in [23], most of the estimations of auto regression parameters prefer the OLS approach. 


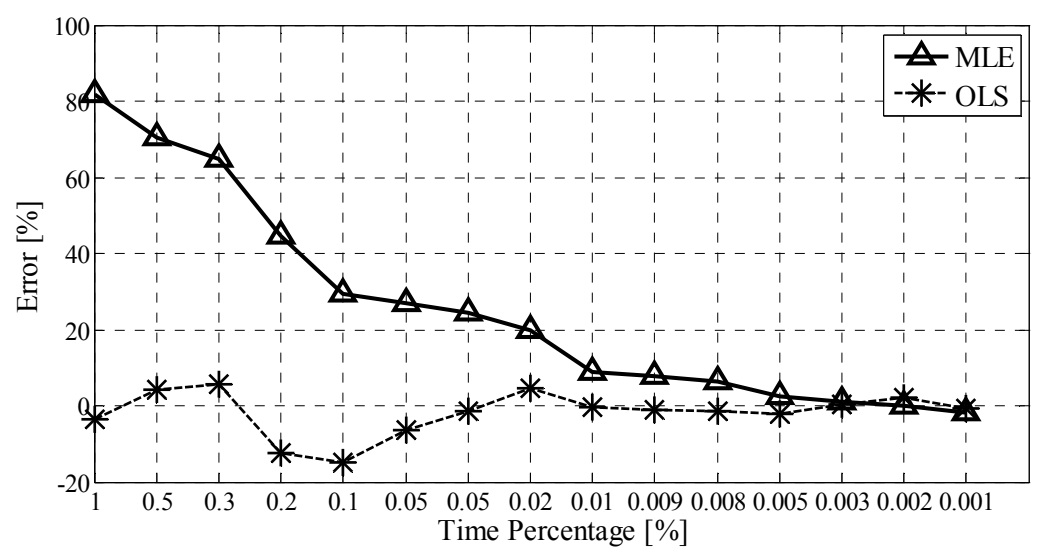

Figure 5. Error percentage comparisons between MLE and OLS method across different time percentage

\section{CONCLUSION}

This work presents a new stochastic approach for the generation of a rain attenuation time series, mainly focusing on the area of extremely heavy precipitation, in particular equatorial Malaysia. This new model employed the well-known CIR model frequently used in economics to reproduce a long-term time series of rain attenuation, the parameters of which were derived by the MLE method and the OLS approach. The model has been compared with a local experimental database in terms of first-order rain attenuation statistics recorded from the MEASAT satellite link. The predicted long-term statistics by the OLS method are found to be in good agreement with the measured attenuation statistics while the MLE tends to overestimate the measurement results. The achieved results offer communication system designers an alternative model utilizing locally derived parameters with better accuracy for the prediction of long-term rain attenuation statistics for satellite channels as well as $5 \mathrm{G}$ cellular channel in heavy rain regions.

\section{ACKNOWLEDGEMENTS}

This work has been funded by Ministry of Education Malaysia and UTM under "Research University Grant" Vot. No. Q.J.130000.2523.07H50 and "HICOE Research Grant" Vot. No. R.J130000.7823.4J221.

\section{REFERENCES}

[1] Felix C., et al., "Ka-band propagation campaign in Malaysia-first months of operation and site diversity analysis," in 10th European Conference on Antennas and Propagation, 2016. EuCAP 2016, pp. 1-5, 2016.

[2] A. D. Panagopoulos, et al., "Satellite communications at $\mathrm{Ku}, \mathrm{Ka}$ and $\mathrm{V}$ bands: propagation impairments and mitigation techniques," IEEE Commun. Surv. Tutorials, vol. 6, pp. 2-14, 2004.

[3] L. Luini and C. Capsoni, "Efficient Calculation of Cloud Attenuation for Earth-Space Applications," IEEE Antennas and Wireless Propagation Letters, vol. 13, pp. 1136-1139, 2014.

[4] L. Luini and C. G. Riva, "Improving the Accuracy in Predicting Water-Vapor Attenuation at Millimeter-Wave for Earth-Space Applications," IEEE Transactions on Antennas and Propagation, vol/issue: 64(6), pp. 2487-2493, 2016.

[5] I. F. Elshami and J. Din, "Seasonal and Diurnal Variation on Tropospheric Scintillation at Ku-Band in Tropical Climate," International Journal of Electrical and Computer Engineering (IJECE), vol/issue: 6(4), 2016.

[6] H. Y. Lam, et al., "Investigation of Rain Attenuation in Equatorial Kuala Lumpur," IEEE Antennas Wireless Propag. Lett, vol. 11, pp. 1002-1005, 2012.

[7] A. I. Yussuff and N. H. Khamis, "Rain Attenuation Modelling and Mitigation in The Tropics: Brief Review," International Journal of Electrical and Computer Engineering, vol/issue: 2(6), pp. 748-757, 2012.

[8] H. Y. Lam, et al., "Statistical and Physical Descriptions of Raindrop Size Distributions in Equatorial Malaysia from Disdrometer Observations," Advances in Meteorology, pp. 1-14, 2015.

[9] T. Maseng and P. M. Bakken, "A Stochastic Dynamic model of Rain attenuation," IEEE Transactions on Communications, vol. 29, pp. 660-669, 1981.

[10] S. Bertorelli, et al., "Generation of Attenuation Time Series for Simulation Purposes Starting From ITALSAT Measurement," IEEE Transactions on Antennas and Propagation, vol/issue: 56(4), pp. 1094-1102, 2008.

[11] J. Lemorton, et al, "Development and validation of time-series synthesizers of rain attenuation for Ka-band and Q/V-band satellite communication systems," International Journal of Satellite Communication Networks, vol. 25, pp. 575-601, 2007. 
[12] ITU-R recommendation P.1853-1, "Tropospheric attenuation time series synthesis," Gene:va, 2012.

[13] C. Guillaume, et al, "A new 'event-on-demand'synthesizer of rain attenuation time series at Ku-, Ka-and Q/V-bands," International Journal of Satellite Communications and Networking, vol. 29, pp. 47-60, 2011.

[14] B. Xavier, et al, "A Rain Attenuation Time-Series Synthesizer Based on a Dirac and Lognormal Distribution," Antennas and Propagation, IEEE Transactions on, vol/issue: 61(3), pp. 1396-1406, 2013.

[15] A. Fernando, et al, "Rain attenuation time series synthesizer based on the gamma distribution," Antennas and Wireless Propagation Letters, IEEE, vol. 10, pp. 1381-1384, 2011.

[16] S. Kanellopoulos, et al, "Satellite and Terrestrial Links Rain Attenuation Time Series Generator for Heavy Rain Climatic Regions," Antennas and Propagation, IEEE Transactions on, vol/issue: 61(6), pp. 3396-3399.

[17] J. C. Cox, et al, "A Theory of the Term Structure of Interest Rate," Econometrica, vol. 53, pp. 385-408, 1985.

[18] P. E. Kloeden and E. Platen, "Numerical Solution of Stochastic Differential Equations," Springer, 1992.

[19] B. Øksendal, "Stochastic Differential Equations: An Introduction with Applications," 6th Edition, Springer, 2010.

[20] Kanellopoulos, et al, "Calculation of the dynamic input parameter for a stochastic model simulating rain attenuation: a novel mathematical approach," Antennas and Propagation, IEEE Transactions on, vol/issue: 55(11), pp. 3257-3264, 2007.

[21] E. Matricciani, "Physical-mathematical model of the dynamics of rain attenuation based on rain rate time series and a two-layer vertical structure of precipitation," Radio Science, vol/issue: 31(2), pp. 281-295, 1996.

[22] G. D. Rossi, et al, "Maximum Likelihood Estimation of the Cox-Ingersoll-Ross Model Using Particle Filters," Computational Economics, vol. 36, pp. 1-16, 2010.

[23] J. D. Hamilton, "Time series analysis," Princeton, Princeton university press, vol. 2, 1994.

[24] E. Matricciani and C. Riva, "The search for the most reliable long-term rain attenuation CDF of a slant path and the impact on prediction models," Antennas and Propagation, IEEE Transactions on, vol/issue: 53(9), pp. 3075-3079, 2005.

[25] O. Vasicek, "An equilibrium characterization of the term structure," Journal of Financial Economics, vol. 5, pp. $177-188,1977$.

[26] S. L. Jong, et al, "Analysis of Fade Dynamic at Ku-Band in Malaysia," International Journal of Antennas and Propagation, 2014.

[27] "Propagation data and prediction methods required for the design of earth-space telecommunication systems," Propagation in non-ionized media. Geneva, Switzerland, ITU-R: Recommendation, pp. 61 8-12, 2015.

\section{BIOGRAPHIES OF AUTHORS}

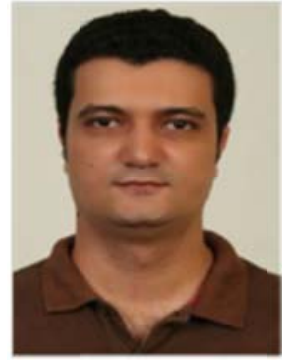

Masoud Mohebbi Nia received his B. S. in Electrical Engineering (Electronics) from Mazandaran University of Iran in 2006, M. Eng. in Communication Engineering and PhD in Electrical Engineering from Faculty of Electrical Engineering (FKE), Universiti Teknologi Malaysia (UTM) in 2011 and 2016 respectively. His research interests include wireless and satellite communication networks, wave propagation, atmospheric issues, Cognitive Radio and green telecommunications service providers as well as High Altitude Platform Station (HAPS).

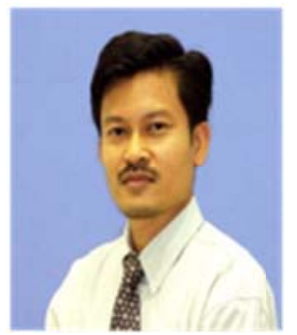

Jafri Bin Din received his BSEE in Electrical Engineering from Tri-State University, Indiana, USA, in 1988 and his Ph.D. from Universiti Teknologi Malaysia (UTM), Johor, Malaysia, in 1997. He has been Head of Departments, Undergraduate Academic Manager and Deputy Dean (Development) in Faculty of Electrical Engineering (FKE), UTM from 2008 till 2013. He is currently the Deputy Director of Wireless Communication Centre at FKE, UTM. His research activities have been correlated to the fields of radio wave propagation, satellite propagation and communications, High Altitude Platform Station (HAPS), satellite TV broadcasting, Weather Radar, and sound techniques for fisheries industry. 


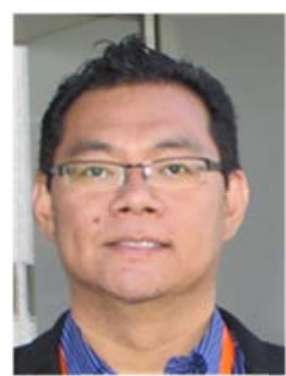

Hong Yin Lam received the M. Eng. and the Ph.D. degrees in electrical engineering and telecommunication from the Universiti Teknologi Malaysia (UTM), Johor, Malaysia, in 2009 and 2013, respectively. He then served as a postdoctoral fellow in the Department of Communication Engineering (UTM), Johor, Malaysia . He is currently a lecturer at Faculty of Engineering Technology, Universiti Tun Hussein Onn, Malaysia, since 2015. His research activities have been relative to the field of radio wave propagation through the atmosphere, with specific focus on rain for propagation applications using meteorological weather radar physical modeling and synthesis of the meteorological environment (precipitation); expected performance of SatCom, and terrestrial wireless links operating in the 10 to100 $\mathrm{GHz}$ range.

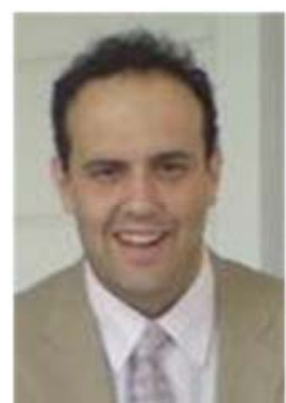

Athanasios D. Panagopoulos was born in Athens, Greece, on January 26, 1975. He received the Diploma degree in Electrical and Computer Engineering (summa cum laude) and the Dr. of Engineering degree from National Technical University of Athens (NTUA) in July 1997 and in April 2002. From May 2008 to May 2013, he was a lecturer in the School of Electrical and Computer Engineering of NTUA, and now he is an assistant profesisor. His research interests include radio communication systems design, wireless and satellite communications networks, the propagation effects on multiple access systems, and on communication protocols. He has participated in ITU-R and ETSI study groups, and he is a member of Technical Chamber of Greece. He is a senior member of IEEE and chairman of the IEEE Greek Communication Chapter. He serves on the editorial boards of Elsevier Physical Communication, and finally, since 2008, he is an associate editor of IEEE Transactions on Antennas and Propagation. 